\title{
Analysis of Effective Length Factors of Hinged Pitched-Roof Framed Column with Sway under Arch Effect
}

\author{
PingLi $^{1 *}$, LipingLiu ${ }^{1}$ and ZunliTeng ${ }^{1}$ \\ ${ }^{1}$ Department of civil engineering, Long Dong University, Qing Yang, Gan Su, 745000, China
}

\begin{abstract}
Aiming at the limitation of the calculated length coefficient of the gable portal rigid frame columns in the standards and regulations, which ignores the influence of the arch effect caused by the axial force of the inclined beams lope, this paper uses the elastic buckling equation of the solid-webbed articulated pitched-roof frame, considered $P-\Delta$ second-order effect and the arch effect of the inclined beam under uniform load together. And the length coefficient calculated is analyzed. It is concluded that the larger the slope of the inclined beam and the ratio of the rigid frame span are, the more obvious the arching effect is, and the larger the calculated length coefficient of the rigid frame column is.
\end{abstract}

\section{Introduction}

For the gable portal light rigid frame, reasonable support and tie bars can ensure the stability of the rigid frame outside-plane. Therefore, the overall stability of the rigid frame is determined by the inside-plane stability. Relevant codes and regulations calculate the stability of the rigid frame through calculating the length coefficient. Under the transverse uniform load, there is an axial force caused by the slope at the joint between the inclined beam and the vertical beam. The articulated gabled portal rigid frame exhibits arch-like characteristics in terms of stress and stability, namely the arch effect. At present, considering the stability of the gable portal frame, the related codes and regulations are to simplify the inclined beam as a horizontal beam. Such simplification fails to consider the influence of arch effect on the overall stability of the rigid frame. This paper will comprehensively consider $P-\Delta$ second-order effect and the arch effect of the inclined beam under the uniform load, and analyze the relationship between the calculated length coefficient and the arch effect.

\section{Elastic buckling equation of rigid frame}

The displacement method is used to solve the elastic buckling equation of the gable portal frame shown in Figure 1. Under the vertical uniform load, the gabled rigid frame has a span change effect [1]. As shown in Fig. 1 , the ridge point $\mathrm{A}$ moves down, the inclined beam pushes the column top outward, and the eaves nodes B and $\mathrm{C}$ move $\Delta$ simultaneously. Even if there is no horizontal load, there will be a second-order $P-$ $\Delta$ effect. This effect that increases the bending moment and displacement is called the span effect[2]. Therefore, the total lateral displacement $\Delta$ of the column is the sum of the lateral displacement $\Delta_{1}$ caused by the bending deformation under the vertical load and the $\Delta_{2}$ generated by the horizontal thrust under the arch effect.

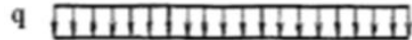

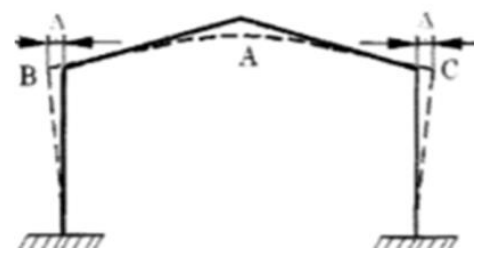

Figure 1. Span variation of pitched-roof frame

The arch effect causes an axial force $N$ at the beam end, as shown in Figure 2, ignoring the small pressure changes caused by the lateral displacement of the column. According to the deformation characteristics of the rigid frame with lateral displacement, the lateral displacement of the ridge node is equal to that of the beam and column nodes at both ends. The pedestal is hinged, the bending moment is 0 , and the ridge point is where the reverse bending point is, so the bending moment is also 0 , and the vertical displacement is also 0 , but there can be side shifts and corners. The reference ${ }^{[2]}$ derived the elastic buckling equation of the pedestal-shaped rigid frame. The equation for the angular displacement of the column insulator shown in the Fig. 2:

\footnotetext{
*e-mail: 253679058@qq.com
} 

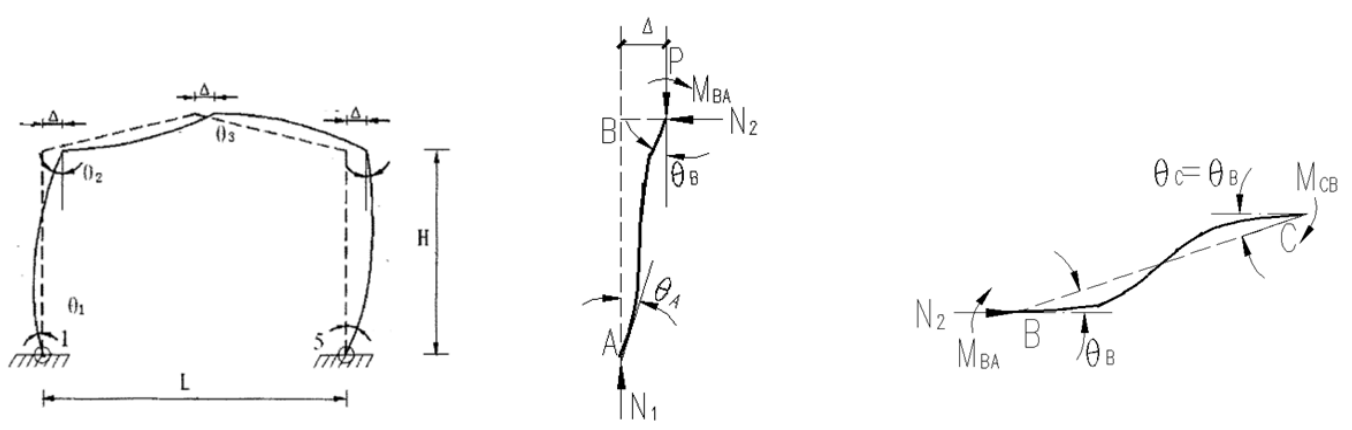

Figure 2. Hinged pitched-roof framed column with sway

$$
\begin{aligned}
& M_{A B}=K\left[C \theta_{A}+S \theta_{B}-(C+S) \Delta / l\right] \\
& M_{B A}=K\left[C \theta_{B}+S \theta_{A}-(C+S) \Delta / l\right]
\end{aligned}
$$

Because the lateral shift angle of the column $\rho=\Delta / l$, $K_{C}=E I_{c} / l_{c}$, according to the boundary condition $M_{A B}=0$, above equation can be written as:

$$
\theta_{A}=-(S / C) \theta_{B}+(1+S / C) \rho
$$

Substituting equation (3) into equation (2), it can be obtained:

$$
M_{B A}=K_{C}\left(C-S^{2} / C\right)\left(\theta_{B}-\rho\right)
$$

The equilibrium equation of the column insulator is: $M_{B A}+P \Delta=0, M_{B A}=-P \rho l_{c}$

$$
-P \rho l_{c}=K_{c}\left(C-S^{2} / C\right)\left(\theta_{B}-\rho\right)
$$

Combining with $P=k^{2} E I_{c}$, Equation (5) can be rewritten as:

$$
\left(C-S^{2} / C\right) \theta_{B}-\left[C-S^{2} / C-\left(k l_{c}\right)^{2}\right] \rho=0
$$

The equation of angular displacement of the beam insulator is:

$$
M_{B C}=K_{b}\left(4 \theta_{B}+2 \theta_{B}\right)-N h_{1}=6 K_{b} \theta_{B}-N h_{1}
$$

$\sum M_{B}=0, \quad M_{B A}+M_{B C}=0$, the buckling equation of the rigid frame is:

$$
K_{c}\left[\left(C-S^{2} / C\right) \theta_{B}-\left(C-S^{2} / C\right) \rho\right]+6 K_{b} \theta_{B}-N h_{1}=
$$

Substituting $\mu=\pi / k l_{c}$ into equation (8), the buckling equation can be rewritten as:

$$
(\pi / \mu) \tan (\pi / \mu)-6 \frac{I_{b} l_{c}}{I_{c} l_{b}}-N h_{1}=0
$$

Through the above derivation, it can be seen that the arch effect exists, which reduces the bending moment acting on the inclined beam and effectively reduces the cross-sectional size of the beam. LinfengLu, QiangGu and QiXie[3] who are using a finite element method analyzed the $P-\Delta$ of the portal frame, and concluded that the rectangular portal frame cannot be simply replaced by a rectangular portal frame. From the above process of calculating the elastic buckling equation, it can be seen that the effect of the $P-\Delta$ effect and arch effect (axial force $\mathrm{N}$ ) on the gable portal frame is obvious. Given the section size of beams and columns, we plug in $\mathrm{C}$ and $\mathrm{S}$, And through repeated trial calculation of formula (9), the $\mu$ value can be calculated to range from 2 to $\infty$.

\section{Calculation of the length factor}

In the design of a rigid frame, the traditional method is to classify the various types of loads acting on the rigid frame, determine the internal force according to the calculation method of structural mechanics, and combine to obtain the most unfavorable internal force of the component. After the maximum bending moment and axial force value of the rigid frame column are determined, the in-plane stability of the rigid frame is calculated according to the bending and bending members. In this design, the reduction of the bearing capacity of the rigid frame by the $P-\Delta$ effect is based on the calculation of the length $l_{0}=\mu l_{c}$ to approximately consider the adverse effects. Among them, the horizontal side shift $\Delta$ of the top of the column-shaped portal frame is actually the sum of $\Delta_{1}$ generated by bending deformation under vertical load and $\Delta_{2}$ generated by horizontal thrust under arch effect. According to the above buckling equation (9) of the gabled rigid frame, the precise value of $\mu$ can be calculate.

"Steel Structure Design Standard" (GB50017-2003) Schedule D-2 gives the inside-plane simplified method (approximate method)[4] of the pedestal-type portal frame. It has been proved in practice that the approximate calculation method of flattening the inclined beam in the standard is unsafe[5].

\section{Calculation results and analysis}

This paper selects the H-shaped equal-section gable portal frame as model. By changing the height and span of the gable portal frame, the slope of the inclined beam, and the beam-column stiffness ratio, the axial force generated by the arch effect is considered to stabilize the gable portal frame as a whole. The calculated length coefficient of the column under the arch effect in the table is calculated by the finite element method. The whole is divided into multiple units of equal cross-section. Three groups of models with different high-span ratios are selected, and the calculated results are compared through looking up table of "Steel 
Structural Design Standard” (GB50017-2017).

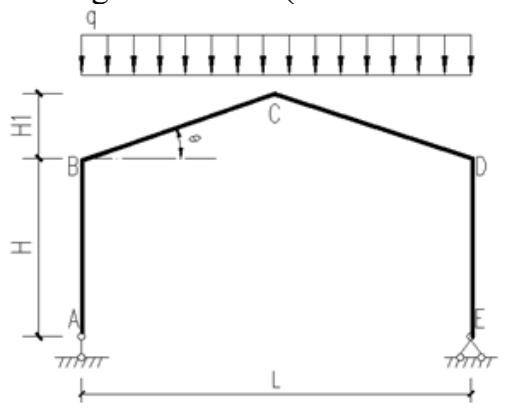

Select the first group of models:

The portal frame has a span of 24 meters, a column height of 6 meters, and a beam column using H $400 \times 200$ $\times 6 \times 12$, as shown in Figure.

Change the slope of the inclined beam, the length of the inclined beam is $L_{b}, \mu$ represents the calculated length coefficient of the rigid frame column considering the influence of the arch effect, $\mu_{0}$ represents the calculated length coefficient of the rigid frame column determined according to the standard[5].

Figure 3. Calculation model

Table 1. Effective length factors of hinged pitched-roof framed column

\begin{tabular}{c|c|c|c|c|c|c}
\hline even load & slope & $L_{b}$ & $i$ & $\mu$ & $\mu_{0}$ & $\frac{\mu-\mu_{0}}{\mu_{0}} \times 100 \%$ \\
\hline \multirow{5}{*}{$6000 \mathrm{~N} / \mathrm{m}^{2}$} & $10^{\circ}$ & 12185 & 0.492 & 3.559 & & 6.23 \\
\cline { 2 - 5 } & $15^{\circ}$ & 12423 & 0.483 & 3.666 & & 9.45 \\
\cline { 2 - 5 } & $20^{\circ}$ & 12770 & 0.470 & 3.781 & & 12.87 \\
\cline { 2 - 5 } & $25^{\circ}$ & 13241 & 0.453 & 3.902 & \multirow{3}{*}{3.35} & 16.49 \\
\cline { 2 - 5 } \cline { 3 - 5 } & $30^{\circ}$ & 13856 & 0.433 & 4.034 & & 20.41 \\
\cline { 2 - 4 } & $35^{\circ}$ & 14649 & 0.410 & 4.179 & & 24.76 \\
\cline { 2 - 4 } & $40^{\circ}$ & 15665 & 0.383 & 4.344 & & 29.67 \\
\cline { 2 - 4 } & $45^{\circ}$ & 16971 & 0.356 & 4.534 & & 35.35 \\
\hline
\end{tabular}

Select the second group of models:

The span of the portal frame and the cross section of the beam and column are unchanged. The height of the column is increased to $8 \mathrm{~m}$. The slope of the inclined beam is changed. The calculated results are shown in Table 3:

Table 2. Effective length factors of hinged pitched-roof framed column

\begin{tabular}{|c|c|c|c|c|c|c|}
\hline even load & slope & $L_{b}$ & $i$ & $\mu$ & $\mu_{0}$ & $\frac{\mu-\mu_{0}}{\mu_{0}} \times 100 \%$ \\
\hline \multirow{8}{*}{$6000 \mathrm{~N} / \mathrm{m}^{2}$} & $10^{\circ}$ & 12185 & 0.667 & 3.415 & \multirow{8}{*}{3.24} & 5.4 \\
\hline & $15^{\circ}$ & 12423 & 0.644 & 3.506 & & 8.2 \\
\hline & $20^{\circ}$ & 12770 & 0.627 & 3.600 & & 11.1 \\
\hline & $25^{\circ}$ & 13241 & 0.604 & 3.703 & & 14.3 \\
\hline & $30^{\circ}$ & 13856 & 0.577 & 3.813 & & 17.7 \\
\hline & $35^{\circ}$ & 14649 & 0.546 & 3.933 & & 21.4 \\
\hline & $40^{\circ}$ & 15665 & 0.511 & 4.072 & & 25.7 \\
\hline & $45^{\circ}$ & 16971 & 0.471 & 4.231 & & 30.6 \\
\hline
\end{tabular}

Select the third group of models:

The span of the portal frame and the cross section of the beam and column are unchanged. The column height is increased to $12 \mathrm{~m}$, and the slope of the inclined beam is changed. The calculation results are shown in Table 4:

Table 3. Effective length factors of hinged pitched-roof framed column

\begin{tabular}{c|c|c|c|c|c|c}
\hline even load & slope & $L_{b}$ & $i$ & $\mu$ & $\mu_{0}$ & $\frac{\mu-\mu_{0}}{\mu_{0}} \times 100 \%$ \\
\hline \multirow{5}{*}{$6000 \mathrm{~N} / \mathrm{m}^{2}$} & $10^{\circ}$ & 12185 & 0.985 & 3.142 & & 4.4 \\
\cline { 2 - 5 } & $15^{\circ}$ & 12423 & 0.966 & 3.212 & \multirow{3}{*}{3.01} & 6.7 \\
\cline { 2 - 5 } \cline { 3 - 5 } & $20^{\circ}$ & 12770 & 0.940 & 3.281 & 9.0 \\
\cline { 2 - 4 } & $25^{\circ}$ & 13241 & 0.906 & 3.362 & & 11.7 \\
\cline { 2 - 4 } & $30^{\circ}$ & 13856 & 0.866 & 3.443 & & 14.4 \\
\hline
\end{tabular}




\begin{tabular}{l|c|c|c|c|c|c}
\hline & $35^{\circ}$ & 14649 & 0.819 & 3.537 & \multirow{5}{*}{} & 17.5 \\
\cline { 2 - 5 } \cline { 3 - 4 } & $40^{\circ}$ & 15665 & 0.766 & 3.642 & & 21.0 \\
\cline { 2 - 4 } & $45^{\circ}$ & 16971 & 0.707 & 3.763 & & 25.0 \\
\hline
\end{tabular}

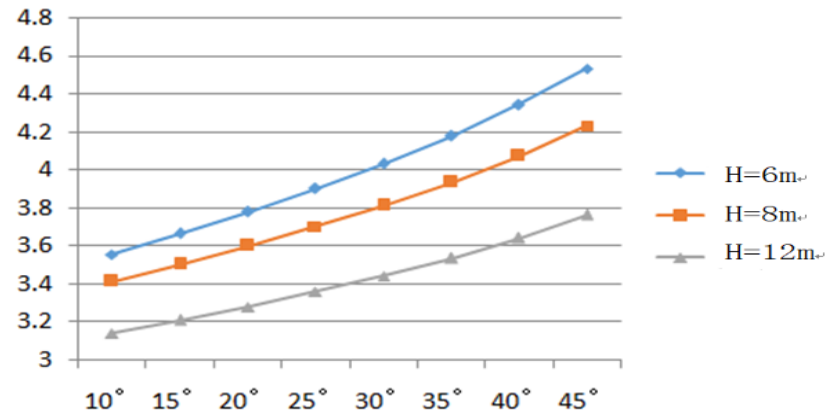

Figure 4. Variation of calculated length coefficient of gable portal frame columns with slope

Analyzing the calculation results in Tables 1, 2, and 3, it can be found that when the slope of the inclined beam is below $20^{\circ}$, the calculated length coefficient $\mu$, which takes into account the influence of the arch effect, is not more than $10 \%$ from the calculated length coefficient $\mu_{0}$, which determined based on the standard[4]. The calculated difference between the two is not large, indicating that within this slope range, the axial force generated at the beam-column connection is very small, and the oblique beam arch effect is not obvious, which can be calculated according to the length of the oblique beam being flattened, and the arch effect can be ignored. However, as the slope increases, the range of the difference between the calculated lengths determined by the two methods also increases. When the slope is $45^{\circ}$, the maximum energy difference between the two is $35 \%$. It can be seen that when the slope is greater than $20^{\circ}$, the axial force at the joint between the inclined beam and the column is larger, and the arch effect is more obvious. It is not safe to treat the length of the inclined beam as straight beam. The internal force of the portal rigid frame calculated by taking the arch effect into consideration is more secure than empirical calculations. The code for the calculation of the length coefficient of the columns of the portal frame is not applicable to that the slope is greater than 1:3[2]. In addition, keeping span of frame, section of member, slope of beam and uniform load the same, the effective length factors of pitched-roof framed column decreases as the column height increases.

\section{Conclusion}

Based on the analysis of the elastic buckling equation of the articulated gable portal rigid frame, this paper considers the arch effect caused by the axial force at the intersection of the inclined beam and the column, and studies the calculated length coefficient $\mu$ of the rigid frame column. The analysis shows that:

At the same slope, the larger the span-height ratio of the rigid frame, the smaller the axial force at the connection between the inclined beam and the column, and the smaller the arch effect on the calculated length coefficient;
The larger the slope, the greater the influence of the arch effect on the calculated length coefficient. When the slope is within $20^{\circ}$, the influence of the arch effect can be negligible. According to the relevant standards and regulations of the pedestal portal frame, the length of the inclined beam should be considered as flat when calculating the length coefficient. When the slope is greater than $20^{\circ}$, the influence of the arch effect is obvious, the calculated length coefficient determined based on standards or procedures need to amend.

\section{Reference}

1. Chen, S.F. (2004) New progress in stability design of steel structures. Progress of steel structures, 12:32-33.

2. Wang, W.F. (2006) Global Stability Analysis of Pitched-Roof Frame Considered Arch Effect under Uniformly Distributed Load. Beijing university of technology, pp. 5-6.

3. Lu, L.F, Gu, Q, Xie Q. (2001) Influences of Portal Frame Roof Pitch on Second-order Effect and Second-order Bending Moment Formula, Building structure, 22:68-70.

4. Design Standard for Steel Structures, GB50017-2017

5. Li, P, Han, G.W. (2008) Influences of Arch Effect on Simply Supported and Tapered Pitched-Roof Framed, journal of north China water resources and hydropower, pp. 43-44. 